\section{LA AUTORREGULACIÓN DE LOS APRENDIZAJES EN ESTUDIANTES UNIVERSITARIOS}

\section{Ana Mercedes Colmenares E.} UPEL-IPB

Recibido: 22-02-06

Aceptado: 17-05-06

\section{Resumen}

Este ensayo se propone la presentación de algunas ideas puntuales, sobre los procesos de aprendizaje y evaluación, esta última desde la perspectiva de la evaluación formadora, caracterizada por fomentar la autorreflexión, autoevaluación y autocorrección permanente en las acciones educativas de los que participan en la construcción de los aprendizajes. Se destaca la autorregulación que plantea la posibilidad real que el estudiante asuma la responsabilidad con respecto a su proceso de aprendizaje, por lo tanto, identifica sus propias debilidades y fortalezas, corrige errores, observa y regula sus actuaciones en las diferentes actividades académicas a las cuales participa, con la colaboración del mediador. Implica para el docente diseñar y transferirles progresivamente las estrategias, propiciar actividades de autorregulación para potenciar dicho proceso; por su parte el alumno debe asumir con verdadera responsabilidad y honestidad sus compromisos académicos y decidir cómo, cuándo y qué aprender.

Palabras claves: autorregulación, evaluación formadora, autonomía

\section{SELF-REGULATION OF LEARNING IN UNIVERSITY STUDENTS Abstract}

This essay proposes the presentation of some ideas about the learning and evaluation process. The latter, from the perspective of forming evaluation, characterised by encouraging permanent selfreflection, self-evaluation and self-correction in the educative actions of the participants in the learning construction. Self-regulation that raises the real possibility that students assume the responsibility about their learning process is highlighted; therefore they identify their own weakness and strengths, correct mistakes, watch and regulate their behaviour in the different academic activities in which he participates with the mediator's cooperation. It implies that the teacher designs and transfers the strategies progressively; encourages self-regulation activities to empower such process; meanwhile students must assume their academic commitments with real responsibility and honesty and saying how, when and what to learn.

Keywords: self-regulation, forming evaluation, autonomy

\section{INTRODUCCIÓN}

El área de estudio del aprendizaje autorregulado ha tenido una expansión de grandes precedentes en los últimos años, sobresalen los planteamientos que vienen realizando autores como: Zimmerman (1990), Sanmartí y Jorba (1995), Monereo (2003), Torranzo y González (2004).

La autorregulación es comprendida como un proceso en el cual el estudiante es capaz de asumir su propia responsabilidad y control sobre su aprendizaje. Dicho proceso se ha estudiado y asumido en tres etapas principales que son: planificación, el monitoreo o control y una fase de reflexión o evaluación.

Ahora bien, la primera etapa conocida como la planificación de actividades de aprendizaje por parte del estudiante, comprende conocer cuáles son los objetivos propuestos, cómo será evaluado, qué se espera de él; en ella activa los conocimientos previos que posee relacionados con el área, tema, contenidos, propuestos. Resulta entonces de vital importancia, que el Docente explique muy bien los objetivos que se propone 
lograr y las expectativas que tiene respecto a los logros y actuaciones de los estudiantes.

En las siguientes etapas, el docente debe propiciar en los estudiantes estrategias para el desarrollo de habilidades metacognitivas que les permita a ellos asumir el control, supervisión y evaluación de sus propios procesos de aprendizaje.

\section{LA AUTORREGULACIÓN DE LOS APRENDIZAJES: UNA VISIÓN PARA EL DESARROLLO DE LA AUTONOMÍA EN LOS APRENDIZAJES.}

El tema de la autorregulación ha sido abordado desde distintas áreas del saber, entre ellas, la psicología, la filosofía y la biología, siendo uno de los campos más fértiles el aprendizaje. Para González citado por Pereira (2001), “La autorregulación implica la modulación del pensamiento, la motivación, la atención y la conducta, mediante la utilización deliberada o automatizada, de mecanismos efectivos y de estrategias de apoyo" (p. 7), por lo tanto será el aprendiz quien elija su proceso educativo, de esta manera se verá en la necesidad de tomar sus propias decisiones, es decir, asumir las riendas de su aprendizaje, afrontando los retos que se le presentan para ir al encuentro de un nuevo conocimiento.

En consecuencia, la autorregulación en el aprendizaje debe ser entendida como la capacidad de la persona para dirigir su propia actuación, por lo tanto, un proceso autorregulado se caracterizará por ser activo, independiente, crítico y reflexivo, contribuyendo a que el individuo alcance su desarrollo pleno, una contínua superación personal y un sentido de autodeterminación en sintonía con la necesidad de educarse permanentemente.
Ahora bien, para que el estudiante desarrolle un aprendizaje autorregulado, según Sanmartí y Jorba (citado por Flores Ochoa 2000), debe aprender al menos tres cosas: identificar los motivos y objetivos del aprendizaje que quiere realizar; anticipar, representarse y planificar las operaciones necesarias para llevar a cabo cada proceso de aprendizaje, seleccionando los procedimientos, estrategias, orden de ejecución y resultados esperados e identificar los criterios de evaluación para saber si las operaciones se desarrollan como estaba previsto y qué correctivos se podrán tomar sobre la marcha.

En referencia a la apropiación de los objetivos de aprendizajes, los autores citados, destacan la necesidad de que el alumno identifique las finalidades del trabajo que realiza, que comprenda por qué se le propician determinadas actividades y por qué se plantean de esa forma. Con respecto a la capacidad de anticipar y planificar acciones, explican que se refiere a la capacidad de representarse mentalmente y explicitar las acciones que deben ejecutar, usando los conocimientos aprendidos para tener éxito en la resolución de tareas que se le proponen.

Por último, la identificación de criterios e instrumentos de evaluación que usa el profesorado debe ser conocido por el estudiante, esto le permite al que aprende activar de manera eficiente los mecanismos de control y regulación.

Desde esta perspectiva, es importante considerar que en todo proceso de aprendizaje están implícitas categorías o variables que pueden favorecer o limitar el proceso en sí. Al respecto, Bandura citado por Pereira (2004), señala que entre las variables determinantes del aprendizaje autorregulado se encuentran las que conciernen al ser como individuo: la motivación, los conocimientos previos y las estrategias de aprendizaje; las referidas a la actuación o conductuales como la auto- 
observación, autoevaluación y la autorreacción y por otro lado las contextuales que corresponden al entorno.

Asimismo, destaca el referido autor, que la autoobservación se refiere a la supervisión sistemática que realiza el individuo de su propia actuación, la autoevaluación implica la comparación con un criterio previo determinado y finalmente la autorreacción se refiere a respuestas que produce el invidividuo ante su propia actuación, aclara también que estos sub-procesos no son secuenciales ni excluyentes.

Atendiendo a las categorías presentadas por Bandura (ob.cit.), con respecto al aprendizaje autorregulado, la orientación que se presenta en este ensayo va dirigido específicamente al desarrollo de los procesos de autoevaluación en los aprendizajes a nivel superior. Tomando en cuenta que el participante es un adulto, que desde el punto de vista cognitivo no puede ser concebido como un ser sin ningún conocimiento, ya que según Pereira (2004) posee "una trama de saberes sobre la cual está cimentada su percepción del mundo y sus diversas interpretaciones", el mismo es capaz de elegir y de permear esa elección con sus emociones más profundas, es en esencia un ser autorregulado.

La autoevaluación ha sido asumida por algunos docentes tradicionales, como una acción trivial e irrelevante, en la cual permiten a los alumnos calificarse las tareas o asignaciones, como un acto magnánimo de congraciarse con ellos, manteniéndolos contentos y animados, pero por supuesto esta práctica no deja aportes significativos que puedan contribuir al desarrollo y formación tanto académica como personal en el estudiante.

Es importante destacar, que la autoevaluación en el sentido verdaderamente pedagógico requiere ciertas condiciones para que tenga sentido formativo y formador, entre las más importantes se puede señalar: que el aprendizaje vaya acompañado de estrategias y procedimientos autorreguladores y que sea monitoreado y permita al estudiante saber en cada fase del proceso cómo va, qué dificultades tiene, cómo resolverlas y si es necesario cambiar de estrategias.

En este sentido, la autoevaluación no será un acto mecánico y de mero cumplimiento de requisitos legales, en el cual el docente presenta en algunos casos un instrumento con criterios e indicadores y el alumno se dedica a cumplir la tarea de rellenar posiblemente en las casillas que se corresponden con la máxima actuación; en otros casos que quizás no constituyan la mayoría, sólo a preguntarle cuánto se merecen, sin atender a ningún parámetro de referencia, sin que exista previo a ello un proceso de reflexión profunda sobre los verdaderos logros que ha alcanzado el estudiante en el marco del curso en el cual se está aplicando esta evaluación.

Al respecto, la autora propone algunas ideas para la aplicación de este proceso de autoevaluación consiste en motivar y orientar al estudiante para que lleve un registro de actividades reflexivas que incluya tanto los aspectos cognitivos como afectivos, después de cada cierto número de sesiones y que puede a su vez compartir con sus pares y con el mediador del curso en entregas negociadas, brindándo la posibilidad de revisar y cambiar aquellas estrategias de aprendizaje que no han sido consideradas como interesantes o significativas, además de los procesos autorreguladores que genera en el alumno, cuando comprende qué cambios debe hacer en sus hábitos de estudio, horarios, tiempo de dedicación, entre otro

El desarrollo de este proceso de manera progresiva puede contribuir significativamente a propiciar o fortalecer la autonomía del estudiante, frente a una dependencia natural 
dentro de los procesos académicos circunscritos en una institución educativa. Por su parte el docente, para ejecutar su práctica evaluativa tiene necesariamente un sentido de dependencia de las normativas legales establecidas y los requisitos administrativos que pautan dicha norma, a su vez tiene autonomía para decidir y negociar con sus alumnos la propuesta de evaluación que desarrollará en su curso.

\section{LA EVALUACIÓN FORMADORA: UNA CONCEPCIÓN DIFERENTE DE ABORDAR LA PRAXIS EVALUATIVA.}

Ahora bien, además de la autoevaluación como forma de participar en la evaluación, bajo la perspectiva constructivista, desde la década de los 90, se viene fortaleciendo una nueva forma de concebir la evaluación planteada por algunos autores, tales como Nunziati (citado por Sanmarti y Jorba, 1995), que se conoce como evaluación formadora, constituye "aquella evaluación en la que la responsabilidad de la regulación recae en el propio estudiante” (p. 35).

Por tanto, va a ser el alumno quien detecte sus errores, reconozca por qué los comete y encuentre sus propios caminos para mejorar, ya que según Astolfi (1999) "el error es la herramienta para aprender, por lo que ocultar las propias concepciones y prácticas o copiar las de otros es lo que más impide aprender, ya que es imposible recibir ayuda para facilitar la autorregulación”. (p. 9).

Esta forma de concebir la evaluación requiere que en los diferentes momentos o etapas del proceso evaluativo, el estudiante tome conciencia de sus debilidades y las posibles causas, sus fortalezas, y además tome decisiones para afrontar las dificultades que se le puedan presentar durante su proceso de aprendizaje, ya que el estudiante con sus compañeros pueden resolver la mayoría de ellas y por supuesto que también cuentan con el apoyo y acompañamiento por parte del profesor.

El docente propicia en el estudiante el desarrollo o mejora de los procesos de autorregulación con su propia actuación, con la interacción, dirigiendo preguntas, anticipando acciones que debe realizar, invitándole a identificar las debilidades en sus producciones, al solicitarle explicación sobre las actividades y cómo éste las ha realizado, de manera que el vaya asimilando e interiorizando procesos de autocontrol, en palabras de Sandonato y Fus (2003),

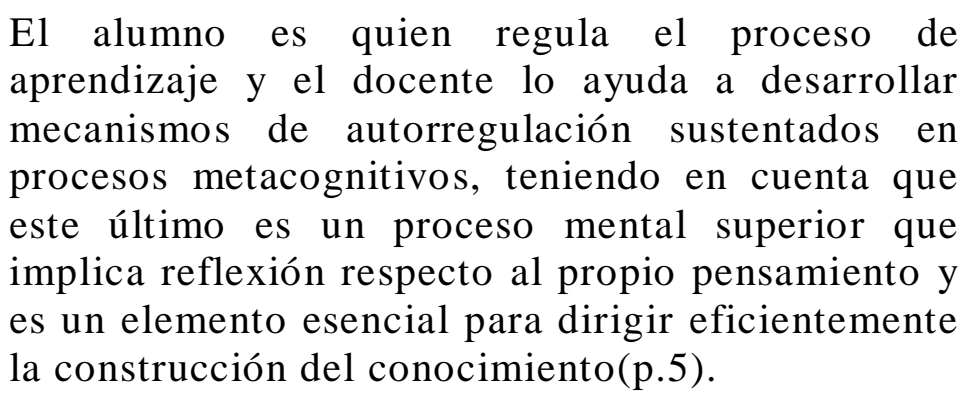
aprendizaje y el docente lo ayuda a desarrollar mecanismos de autorregulación sustentados en procesos metacognitivos, teniendo en cuenta que este último es un proceso mental superior que implica reflexión respecto al propio pensamiento y es un elemento esencial para dirigir eficientemente la construcción del conocimiento(p.5).

De esta manera, el estudiante en situaciones sucesivas pondrá en práctica estrategias autorreguladoras que se evidenciaran por medio del uso de corrección, cuestionamiento, búsqueda de información y anticipación: a medida que se le pide que explique, prediga, verbalice o indique aspectos de las asignaciones, ocurriendo así el proceso de exteriorización, explícito y más consciente de su forma de aprender. Martí ( citado por Flores 2000).

La evaluación formadora, permite que el estudiante logre aprendizajes altamente significativos, por lo tanto, los resultados alcanzados en las diferentes estrategias de evaluación deben ser mucho mejores, por supuesto, que estos logros van a depender según Black y Harrison citado por Sanmarti y Jorba (1995), del 
tipo de actividades que se hayan propuesto y cómo se aplicaron a lo largo del proceso de enseñanza, haciendo énfasis en la forma de plantear las preguntas y de las respuestas como potenciales insumos.

Desde esta perspectiva, es visto el alumno como un ser capaz de autorregularse, para ello es necesario compartir criterios de evaluación, así como también favorecer un clima de armonía y confianza para que el estudiante sea capaz de manifestar sus ideas sin temor a la crítica, a la descalificación, a la comparación con sus pares; además es fundamental que comparta, discuta y argumente sus puntos de vista frente a las diferentes situaciones de aprendizaje.

Para lograr los propósitos de la evaluación formadora, se requiere proponer en el aula nuevas maneras de abordar el aprendizaje y para ello una de las alternativas la constituye el aprendizaje cooperativo, que según David y Roger Jonson, psicólogos sociales, citados por Rue (2005), lo han definido como “aquella situación de aprendizaje en las que los objetivos de los participantes se hallan estrechamente vinculados, de tal manera que cada uno de ellos sólo puede alcanzar sus objetivos si y sólo sí los demás consiguen alcanzar los suyos”. (p.1).

Este tipo de aprendizaje requiere que el docente organice una serie de procedimientos de enseñanza, que incluyan la organización de la clase en pequeños grupos mixtos y heterogéneos, donde los alumnos trabajen conjuntamente de forma coordinada entre sí para resolver tareas académicas y profundizar en el aprendizaje.

Es conveniente destacar, que para promover cambios significativos en la evaluación, es fundamental que se impulsen también a nivel de la enseñanza y para ello es propicio que cómo docentes nos hagamos las siguientes interrogantes: qué se enseña, qué actividades se realizan y cómo se organizan, cómo se atiende a la diversidad de estudiantes, cómo nos relacionamos con ellos y al responder estaremos dando cuentas del modelo de enseñanza que estamos propiciando en nuestras aulas de clase, porque a decir de Flores Ochoa (2000) "Enseñar, aprender y evaluar son tres procesos inseparables, no se puede cambiar uno sin cambiar los demás”. (p. 9).

\section{REFLEXIONES FINALES.}

Las formas de concebir las funciones de la evaluación y su respectiva aplicación, están íntimamente relacionadas con las concepciones sobre la ciencia, por tanto, una visión de ciencia " verdad " conlleva a evaluar si los alumnos saben reproducirla, una visión de la génesis de la ciencia como resultado de la aplicación de procesos racionales de tipo inductivo o deductivo, implica centrar la evaluación en los procesos y por último una visión de la ciencia como construcción social de modelos explicativos, significa concebir la evaluación como proceso de regulación. (Sanmarti y Jorba, 1995).

El proceso de autorregulación en la educación abre las puertas hacia la formación de un alumno activo, emprendedor y verdadero protagonista de su aprendizaje, un participante capaz de: tomar parte en la elección de sus objetivos, metas y tareas, así como también las vías y procedimientos a utilizar para alcanzarlas, aprender a aprender, aprender a reaprender y aprender a desaprender con autonomía, construir caminos individuales al conocimiento, dándole sentido y significado propio a lo que aprende. Todo esto le permitirá dejar atrás los viejos prejuicios reduccionistas, con posturas de aprendizaje 
receptivo, memorístico y pasivo, generados en los esquemas de la educación tradicional y la enseñanza directiva; en palabras de Pereira (2004) esto significa "Romper el cascarón, atreverse a emerger en medio del caos, de la incertidumbre, integrar procesos que connotan aspectos de orden y desorden” (p. 112).

Una experiencia que la autora desea compartir es la práctica consecutiva de un verdadero proceso de autoevaluación, donde se le orienta al estudiante la importancia de asumir sus propios procesos de autorreflexión con miras a tomar conciencia de sus limitaciones, debilidades, potenciales, habilidades, a fin de mejorar o fortalecer aquellos aspectos que le resulten verdaderos obstáculos para alcanzar las metas propuestas. La misma, ha sido realizada a través del uso consecuente de instrumentos de evaluación, especialmente el diario reflexivo que se sugiere al estudiante para que registre sus impresiones después de cada sesión de clase y que comparte con la facilitadora en sesiones posteriores. En conversaciones formales con los estudiantes han evidenciado la importancia de estos procesos reflexivos y el aporte que ello ha significado para reconocer qué deben mejorar.

Para concluir, la invitación es a transitar este nuevo sendero, que si bien es cierto representa un verdadero reto para el docente de este milenio, es también una hermosa oportunidad de darle concreción a estos enfoques contemporáneos que privilegian la participación, la negociación y el consenso, en la praxis evaluativa, potenciando el rol protagónico que significa para el estudiante asumir su propia responsabilidad en los procesos de aprendizaje y evaluación; procesos siempre inacabados de la construcción del modo personal de aprender y que implica el desarrollo de competencias de autorregulación.
Astolfi, J.(1999). El error: un medio para enseñar. Colección Investigación y enseñanza. $\mathrm{N}^{\circ} 15$. Sevilla: Diada Editora.

Flores Ochoa, R. (2000). Autorregulación, metacognición y evaluación. Acción Pedagógica [Revista en línea] Disponible: http://www.saber.ula.ve.

Morín, E. (1999). La cabeza bien puesta. Repensar la reforma. Reformar el pensamiento. Argentina: Ediciones Nueva Visión.

Morín, E. (2001). Introducción al Pensamiento Complejo. España: Gedisa Editor.

Pereira, L. (2004). La autorregulación como proceso complejo en el aprendizaje del individuo peninsular. [Documento en línea] Disponible: http://www.redalyc.uamax.max/rodalyc.

Pozo, I. y Monreo, C. (2002). El aprendizaje estratégico. Enseñar a aprender desde el currículo. España: Santillana.

Rue, J. (2005). Qué es el aprendizaje cooperativo. Instituto de Ciencias de la Ecuación (ICE). [Documento en Línea.] Disponible en http//giac.upc.es/pag/giac_cas/giac_que.es.htm

Sandonato, A; Fus, M (2003). Reflexiones sobre la organización del Currículo de Biología en Base a la Autorregulación de los Aprendizajes. [Documento en Línea.] Disponible en http://wwwensino.univates.br/4iberoamericano/trabalhos/trabakho205.pdf.

\section{REFERENCIAS}


Sanmartí, N y Jorba, J.(1995). Autorregulación en los procesos de aprendizaje y construcción de conocimientos. Alambique. España. 